\title{
Multiple microcrystal deposition within a family
}

\author{
MICHAEL DOHERTY AND PAUL A DIEPPE \\ From the University Department of Medicine, Bristol Royal Infirmary, Bristol
}

SUMmARY A family is described in which four members in three generations showed evidence of: crystal deposition disease: two developed calcium pyrophosphate dihydrate (CPPD) crystaf5 deposition, one calcific periarthritis, and one mixed crystal deposition disease (gout $+\frac{0}{2}$ chondrocalcinosis). This previously undescribed observation supports a possible role for non specific heritable connective tissue factors in predisposing to crystal deposition.

Key words: chondrocalcinosis, calcific periarthritis, gout, pyrophosphate arthropathy, calciumf pyrophosphate dihydrate crystal deposition disease, familial crystal deposition, mixed crystaf deposition.

Familial predisposition has been reported independently for monosodium urate monohydrate (MSUM) crystal deposition in gout, ${ }^{1}$ calcium pyrophosphate dihydrate (CPPD) crystal deposition in pyrophosphate arthropathy, ${ }^{2}$ and carbonated apatite (basic calcium phosphate) deposition in idiopathic chondrocalcinosis ${ }^{3}$ and calcific periarthritis. ${ }^{4}$ The mechanisms of crystal deposition in these diseases, however, are poorly understood, and the genetic factors that enhance susceptibility have not been determined.

We report a family in which all three of the major crystal deposition diseases are represented in different family members. Such an observation supports a role for non-specific heritable connective tissue factors concerned with crystal nucleation and growth in predisposing to crystal deposition diseases.

\section{Case reports}

The index case was an 83-year-old Caucasian woman (patient 1) who presented with chronic bilateral gonarthrosis. Symptoms had started 30 years before but had worsened and become persistent over the previous five years. Examination showed a fit woman with no evidence of systemic disease. Both knees were stable but showed varus deformity, quadriceps wasting, patellofemoral cre-

Accepted for publication 4 March 1985.

Correspondence to Dr M Doherty, Consultant Senior Lecturer, Rheumatology Unit, City Hospital, Hucknall Road, Nottingham NG5 1PB. pitus, effusion, and restricted flexion. Addition $\vec{x}$ joint involvement included bilateral Heberden' $\%$ nodes, non-tender enlargement of both second an $\$$ third metacarpophalangeal joints, 'squaring' of bott first carpometacarpal joints, restricted movemen with crepitus of right shoulder and left subtalaô joints, and bilateral hallux valgus. Fluid aspirate from both knees was viscous, with total white cel counts of $18000 / \mathrm{mm}^{3}\left(18 \times 10^{9} / 1\right), 70 \%$ neutrophil\$ (right knee) and $16000 / \mathrm{mm}^{3}\left(16 \times 10^{9} / \mathrm{l}\right), 60 \%$ neu trophils (left knee); polarised microscopy in eact. instance showed triclinic crystals $2-10 \mu \mathrm{m}$ in length with positive birefringence and inclined extinction consistent with CPPD. Knee radiographs showe bilateral chondrocalcinosis with changes typical of pyrophosphate arthropathy (Fig. 1). Changes of pyrophosphate arthropathy, without chondrocalci nosis, were also marked in both shoulders an $\$$ present in all other clinically involved joints; chon drocalcinosis as an isolated abnormality was present in the left acromioclavicular joint and in both wrist triangular ligaments. Radiographs of thoracolumbar spine and pelvis showed only moderate spondylosiso Laboratory investigations included normal fulo blood count and plasma viscosity: serum was nega? tive for rheumatoid and antinuclear factors, and screening of serum calcium, phosphate, alkalin phosphatase, urea, magnesium, iron, uric acid, sugar, and thyroid function excluded underlying metabolic or endocrine disease.

The diagnosis was primary generalised osteoar thritis with pyrophosphate arthropathy. Symptoms and mobility were improved by joint aspiration 

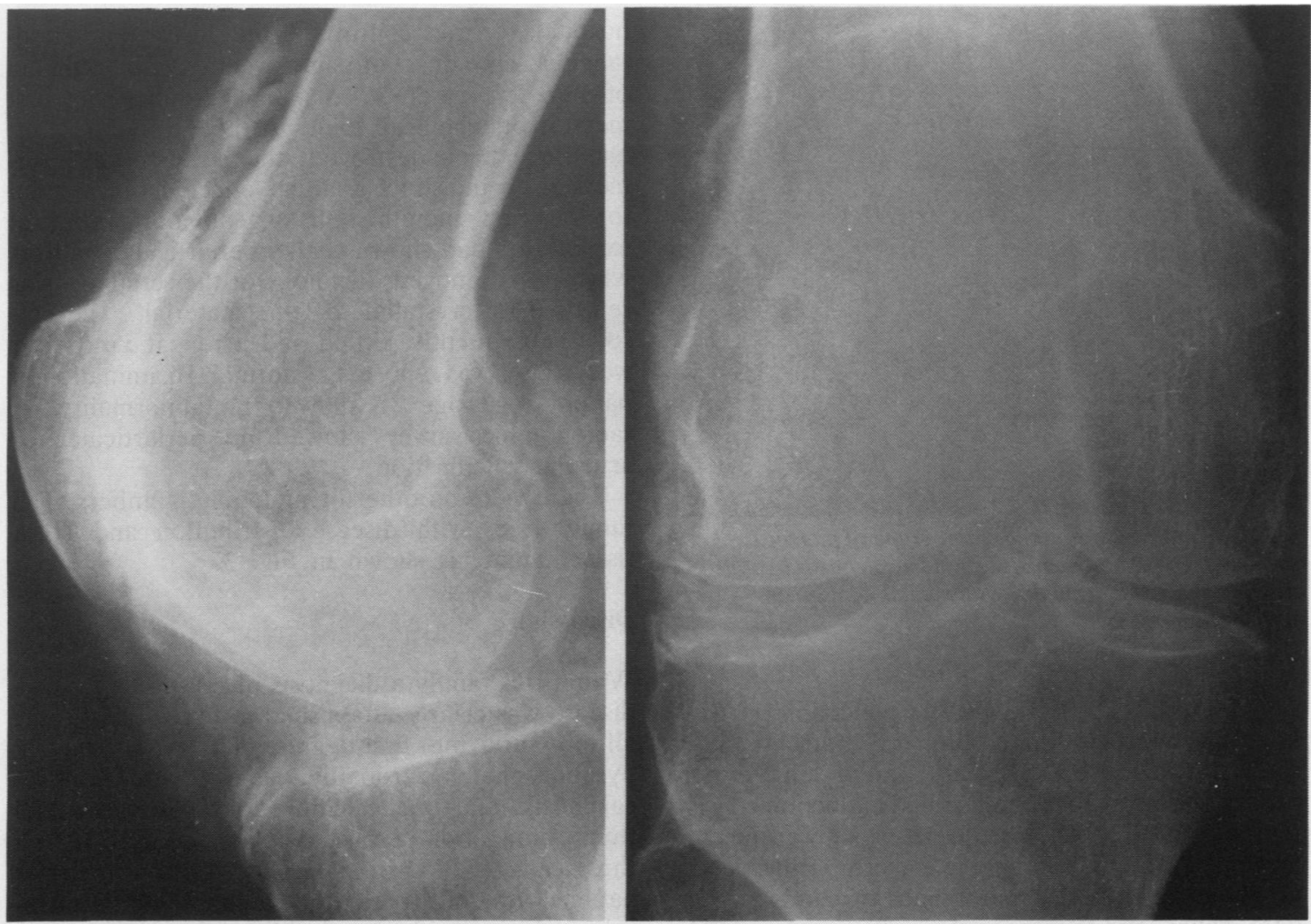

Fig. 1 Lateral and anteroposterior knee radiograph of index case (patient 1) showing calcification of hyaline and meniscal cartilage, with joint space narrowing, sclerosis, and exuberant new bone formation, predominantly affecting the patellofemoral compartment.

physiotherapy, and analgesic anti-inflammatory drugs.

Enquiry into the family history suggested a high incidence of joint disease with symptoms in her son, one daughter, and her only grandchild (her one other daughter had complained of no joint symptoms and had died of cancer at age 47). Surviving family members were therefore contacted and investigated.

Patient 1's 67-year-old son (patient 2) had a 24year history of classic acute gout affecting at various times both first metatarsophalangeal joints, knees, ankles, and right wrist. One knee and one ankle had been aspirated during acute episodes and had shown sterile fluid with needle-shaped negativebirefringent crystals consistent with MSUM. Hyperuricaemia had been repeatedly observed despite successive treatments with sulphinpyrazone $600 \mathrm{mg}$ daily (eight years) and allopurinol $300 \mathrm{mg}$ daily (five years). At age 66 he had developed a lymphoma associated with an IgM paraprotein, for which he received combination chemotherapy. Examination showed an ill man with florid lymphadenopathy: there were no tophi and no evidence of chronic arthropathy. Knee radiographs showed bilateral chondrocalcinosis with medial joint space narrowing (Fig. 2); radiographs of hands, pelvis, and shoulders were normal. Serum urate was $0.6 \mathrm{mmol} / 100 \mathrm{ml}$ (normal $0 \cdot 12-0.46 \mathrm{mmol} / \mathrm{l}$ ), serum urea and electrolytes were normal, and biochemical screening showed no additional metabolic or endocrine disease. Because of his poor general condition knee aspiration was not attempted.

Patient 1's 59-year-old-daughter (patient 3) had a five-year history of intermittent pain, stiffness and swelling of both knees. Examination showed joint line tenderness, effusion, and restricted flexion of both knees; there was no local or generalised hypermobility, and the remainder of the examination was normal. Knee aspiration yielded viscous fluids with total white cell counts of $17000 / \mathrm{mm}^{3}$ $\left(17 \times 10^{9} / 1\right), 60 \%$ neutrophils (right knee) and 


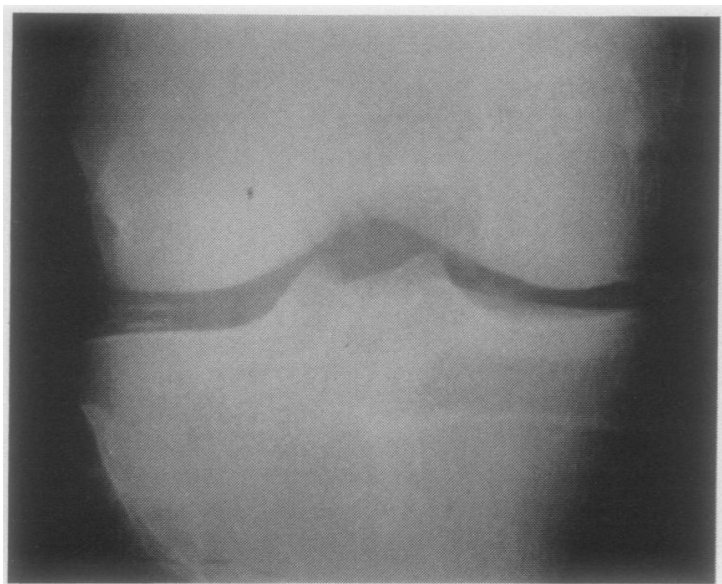

Fig. 2 Anteroposterior knee radiograph of patient 2 showing hyaline and meniscal chondrocalcinosis in both tibiofemoral compartments, with medial joint space narrowing.

$12000 / \mathrm{mm}^{3} \quad\left(12 \times 10^{9} / \mathrm{l}\right), \quad 75 \%$ neutrophils (left knee); polarised microscopy of both samples showed triclinic crystals $4-10 \mu \mathrm{m}$ in length with positive birefringence and inclined extinction consistent with CPPD. Knee radiographs showed medial and patellofemoral compartment osteoarthritis but no chondrocalcinosis; radiographs of hands, shoulders, pelvis, and thoracolumbar spine were normal. Laboratory screening showed no associated metabolic or endocrine disease.

Patient 3's 32-year-old daughter (patient 4) had a history of acute 'gout' at age 12. Examination of hospital records confirmed the history of spontaneous acute onset of pain, swelling, and erythem $\overrightarrow{\vec{F}}$ of her left forefoot, maximal around the first metatarsophalangeal joint. Although radiograph had since been destroyed, the $x$-ray report com $\bar{x}$. mented on indistinct granular opacification media to the first metatarsophalangeal joint. Surgicas exploration had shown chalky periarticular detritus which was removed; histology of the scrapings wa $\vec{\nabla}$ reported as 'crystalline calcific material'. The epi sode subsequently settled and a repeat $x$-ray fous weeks later was reported as normal. Examination of patient 4 at age 32 showed no abnormality. \& radiographic survey showed no periarticular of articular calcification.

There were no other living family members. The family tree, with disease distribution and HLA tissue typing, is shown in Fig. 3.

\section{Discussion}

Within this family a diagnosis of crystal deposition disease was clearly established in four of five (i.e $e_{\infty}^{\infty}$ all four surviving) first degree relatives, two having pyrophosphate arthropathy, one gout with chondrog calcinosis, and one calcific periarthritis. Synovia fluid examination permitted crystal identification i three cases (CPPD in two, MSUM in one). In the patient with gout (patient 2) radiographic chondro\& calcinosis was evidence of additional crystal deposition, most probably of CPPD or basic calcium phosphate. ${ }^{5}$ Pyrophosphate arthropathy without chondrocalcinosis is well described ${ }^{6}$ and does not detract from the diagnosis in the case of patient 3
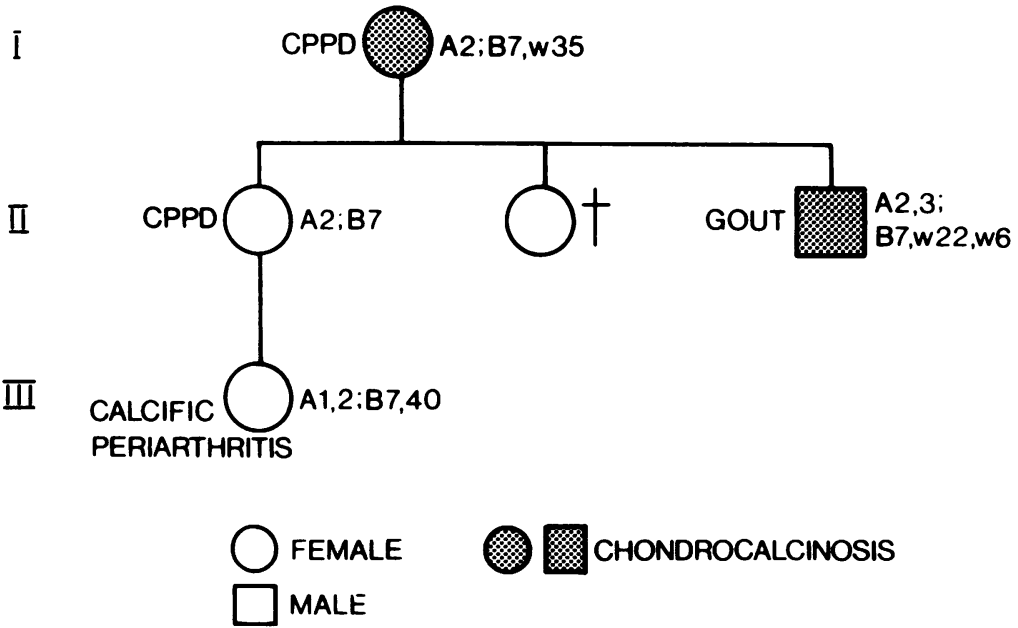

Fig. 3 Pedigree of family showing HLA phenotype and disease distribution. 
Although the diagnosis of calcific periarthritis in the youngest family member (patient 4) was necessarily presumptive, the recorded clinical, radiographic, and histological findings are characteristic of the condition, and no other diagnosis seems tenable. It would therefore appear that all three major crystal deposition diseases are represented within this family. As far as we are aware this is the first report of such an occurrence. Although familial clustering of these conditions could have arisen by chance, the rarity of calcific periarthritis and hereditary CPPD deposition suggests more than coincidental association.

Although hereditary predisposition has been described independently for gout, ${ }^{1}$ CPPD crystal deposition, ${ }^{2}$ calcific periarthritis, ${ }^{4}$ and idiopathic chondrocalcinosis, ${ }^{3}$ the familial factors that lead to crystal deposition in these conditions have not been fully defined. Heritable abnormalities of metabolism that lead to solute excess have been clearly shown in gout ${ }^{7}$ and reported in familial pyrophosphate arthropathy. ${ }^{8}$ The possibility that additional, non-specific crystal promoting factors may also have a role, however, is suggested by the low incidence of gout in hyperuricaemic subjects, ${ }^{9}$ the increased association between gout and chondrocalcinosis, ${ }^{10}$ the occurrence of mixed deposition of different crystal species within individuals, ${ }^{11}$ and the negative association between rheumatoid disease and both MSUM $^{12}$ and CPPD ${ }^{13}$ deposition.

The importance of factors with inhibitory or promoting effects on crystallisation has particularly been emphasised in connection with normal bone mineralisation and dentition, ${ }^{14}$ urolithiasis ${ }^{15}$ gall stone formation, ${ }^{16}$ salivary calculi, ${ }^{17}$ and pancreatic calcification. ${ }^{18}$ In articular tissues factors incriminated in the control of crystal nucleation and growth, particularly of apatite, include inorganic pyrophosphate, matrix vesicles, collagens, mitochondria, acidic phospholipids, $\gamma$-carboxyglutamic acid (GLA)-containing protein, and proteoglycan aggregates. $^{19-21}$ There is little direct evidence, however, of genetic variability in these factors to account for disease susceptibility. Familial variation in local tissue factors, reflected by uronic acid turnover, has been proposed as a major determinant of MSUM deposition in primary gout, ${ }^{22}$ and abnormality of matrix proteoglycan has been reported to predate CPPD deposition in familial pyrophosphate arthropathy. ${ }^{23}$ Facilitation of CPPD deposition by unidentified factors that accompany joint damage and repair is suggested by frequent association with osteoarthritis and by occurrence of localised CPPD deposition in situations of previous joint injury. ${ }^{13} 24$ Similar predisposition to MSUM crystal deposition could in part explain the preferential involvement of Heberden's nodes and osteoarthritic interphalangeal joints in diuretic-induced gout ${ }^{25}$ and predilection for the first metatarsophalangeal joint (the earliest and most common site of radiographic osteoarthritis) in primary disease. ${ }^{26}$ Although positive association between crystal deposition diseases might be explained by epitaxial phenomena, further investigation of factors that promote or inhibit crystal deposition would seem warranted.

The importance of 'soil' as well as 'seed' is further supported by the present observation of multiple deposition of chemically dissimilar crystals within individuals of the same family. In this situation heritable connective tissue factors may be important in permitting development of disease, whereas interaction with additional familial or environmental factors (e.g., hyperuricaemia, generalised osteoarthritis, trauma) may determine the nature and distribution of the crystal deposit.

\section{References}

1 Grahame R, Scott J T. Clinical survey of 354 patients with gout. Ann Rheum Dis 1970; 29: 461-8.

2 Zitnan D, Sitaj S. Chondrocalcinosis articularis. 1. Clinical and radiological study. Ann Rheum Dis 1963; 22: 142-52.

3 Marcos J C, De Benyacar M A, Garcia-Morteo O, et al. Idiopathic familial chondrocalcinosis due to apatite crystal deposition. Am J Med 1981; 71: 557-64.

4 Hajiroussou V J, Webley M. Familial calcific periarthritis. Ann Rheum Dis 1983; 42: 469-70.

5 McCarty D J, Hogan J M, Gatter R A, Grossman M. Studies on pathological calcification in human cartilage. I. Prevalence and types of crystal deposits in the menisci of two hundred and fifteen cadavera. J Bone Joint Surg 1966; 48A: 309-25.

6 Utsinger P D, Zvaifler N J, Resnick D. Calcium pyrophosphate dihydrate deposition disease without chondrocalcinosis. J Rheumatol 1975; 2: 258-64.

7 Seegmiller J E. Human aberrations of purine metabolism and their significance for rheumatology. Ann Rheum Dis 1980; 39: 103-17.

8 Lust G, Faure G, Netter P, Gaucher A, Seegmiller J E. Evidence of a generalized metabolic defect in patients with hereditary chondrocalcinosis: increased inorganic pyrophosphate in cultured fibroblasts and lymphoblasts. Arthritis Rheum 1981; 24: 1517-21.

9 Fessel W J, Siegelaub A B, Johnson E S. Correlates and consequences of asymptomatic hyperuricaemia. Arch Intern Med 1973; 132: 44-54.

10 Hollingworth P, Williams P L, Scott J T. Frequency of chondrocalcinosis of the knees in asymptomatic hyperuricaemia and rheumatoid arthritis: a controlled study. Ann Rheum Dis 1982; 41: 344-6.

11 Dieppe P A, Doyle D V, Huskisson E C, Willoughby D A, Crocker P A. Mixed crystal deposition disease and osteoarthritis. $B r$ Med $J$ 1978; i: $150-1$.

12 Wallace D J, Klinenberg J R, Morhairn D, Berlanstein B, Biren P C, Callis G. Coexistent gout and rheumatoid arthritis: case report and literature review. Arthritis Rheum 1979; 22: 81-6.

13 Doherty M, Dieppe P A, Watt I. Low incidence of calcium pyrophosphate dihydrate crystal deposition in rheumatoid arthritis with modification of radiographic features in coexistent disease. Arthritis Rheum 1984; 27: 1002-9. 
14 Hay D I, Moreno E C, Schlesinger D H. Phosphoprotein inhibitors of calcium phosphate. Precipitation from salivary secretion. Inorg Perspect Biol Med 1979; 2: 271-85.

15 Fleisch $\mathrm{H}$. Inhibitors and promoters of stone formation. Kidney Int 1978; 13: 361-71.

16 Gollish S H, Burnstein M J, Ilson R G, Petrunka C N, Strasbery $S \mathrm{M}$. Nucleation of cholesterol monohydrate crystals from hepatic gall-bladder bile of patients with cholesterol gall stones. Gut 1983; 24: 836-44.

17 Schlesinger D H, Hay D I. Complete covalent structure of statherin, a tyrosine-rich acidic peptide which inhibits calcium phosphate precipitation from human parotid saliva. J Biol Chem 1977; 252: 1689-95.

18 De Caro A, Lohse J, Sarles H. Characterisation of a protein isolated from pancreatic calculi of men suffering from chronic calcifying pancreatitis. Biochem Biophys Res Commun 1979; 87: 1176-82.

19 Vogel J J, Boyan-Salyers B D. Acidic lipids associated with the local mechanism of calcification. A review. Clin Orthop 1976; 118: $230-41$.
20 Malemud C J, Moskowitz R W. Physiology of articula cartilage. In: Hasselbacher $P$, ed. Clinics in rheumatic disease Philadelphia: Saunders, 1981; 7: 29-55.

21 Wuthier R E. A review of the primary mechanism of endochor dral calcification with special emphasis on the role of cells mitochondria and matrix vesicles. Clin Orthop 1982; 16\% 219-42.

$22 \mathrm{Katz}$ W A. Deposition of urate crystals in gout. Altere更 connective tissue metabolism. Arthritis Rheum 1975; 18 (suppl)

23 Bjelle A O. Morphological study of articular cartilage in pyrophosphate arthropathy. (Chondrocalcinosis articularis or calcium pyrophosphate dihydrate crystal deposition disease Ann Rheum Dis 1972; 31: 449-56.

24 Doherty M, Watt I, Dieppe P A. Localised chondrocalcinosis post-meniscectomy knees. Lancet 1982; i: 1207-10.

25 Simkin P A, Campbell P M, Larson E B. Gout in Heberden's nodes. Arthritis Rheum 1983; 26: 94-7.

26 Simkin P A. The pathogenesis of podagra. Ann Intern Meg 1977; 86: 230-3. 